\title{
Improving Rocchio with Weakly Supervised Clustering
}

\author{
Romain Vinot and François Yvon \\ GET/ENST, 46 rue Barrault, \\ 75634 Paris Cedex, France \\ \{romain.vinot, francois.yvon\}@enst.fr
}

\begin{abstract}
This paper presents a novel approach for adapting the complexity of a text categorization system to the difficulty of the task. In this study, we adapt a simple text classifier (Rocchio), using weakly supervised clustering techniques. The idea is to identify sub-topics of the original classes which can help improve the categorization process. To this end, we propose several clustering algorithms, and report results of various evaluations on standard benchmark corpora such as the Newsgroups corpus.
\end{abstract}

\section{Introduction}

The automated categorization of documents 16 into predefined classes has progressively emerged as one of the most popular task in the area of Text Mining technologies. The categorization paradigm provides a very general framework for many practical applications such as filtering, routing, indexation, tracking... Historically, research in automated text categorization was developped promoted by the Information Retrieval community, in the context of text indexation. In the past five to ten years, this task has been rediscovered by the Machine Learning community and has since been the subject of many empirical studies [20], demonstrating the applicability and efficiency of Machine Learning algorithms such as Support Vector Machines [6] and Boosting techniques [14.

The Rocchio algorithm [12], originally proposed as a means to improve information retrieval (IR) systems, is conceptually one of the simplest text categorization algorithm. As such, it has been shown to be, in many experimental conditions, less successful than other approaches, such as SVMs or $k$-NN. Various improvements of this algorithm have been proposed, eg. in [17] [1] 15] or [9], which have been effective in increasing the performance of this methodology.

As discussed for instance in [19], Rocchio is especially well suited in contexts for applications where (i) the number of classes is high; (ii) the n-best answers (rather than just the first one) are taken into account and (iii) class labels are noisy. This seems to happen in practical applications, especially when categories cannot be directly linked with the thematic content of documents. Conversely, we have experimentally demonstrated that classes with heterogeneous content can badly impair its performance. This behaviour is in line with the simplicity of the underlying statistical model and of the learning procedure. 
In this paper, we investigate several procedures allowing Rocchio to automatically adapt its complexity to the data using a weakly supervised clustering. The idea is to identify useful subclasses in the training data, and to use these to refine the decision surface; each new subclass increasing the overall model complexity. The main novelty of this work lies in our attempt to discover these subclasses in such a way that the resulting partition actually improves the final decision rule.

The idea of using unsupervised clustering to improve supervised classification is not entirely new, and has already been suggested in several contexts. In the context of the $k$-nearest neighbors algorithm, many authors have advocated unsupervised clustering as a means to organise very large instance sets, thus speeding up the $k$-nn computation.

In the context of the (TREC) batch filtering task, 4] and 11] incrementally cluster incoming data into subclasses of the original classes. These clusters are then used as (new) regular class labels : any document falling into cluster $S$ of class $C$ is eventually labelled as $C$. Both papers use rather different classification algorithms (Rocchio for the former paper, and SVM for the latter), but neither yield fully conclusive results. In any case, this optimisation does not seem to increase overall performance. One should note that in this approach, clustering and classification are viewed as two separate and unrelated processes.

The motivations of [3] for using unsupervised clustering are clearer: these authors explicitely aim at structuring an heterogeneous corpus of training texts, in the context of a filtering task. Relevant texts are first partitionned into $N$ clusters, using autoclass [2]. Test documents are then classified as follows: for each subclass a relevance judgement is separately computed; these judgements are then linearly combined, using a perceptron. This procedure seems to provide the authors an effective means to isolate those clusters which provide the most relevant judgements.

The idea of [7] is to use clustering techniques in order to make the $k$-nn decision rule less sensible to noisy data, and more like a linear classifier. Proceeding bottom-up, their algorithm recursively aggregates training instances, subject to the condition that (i) they belong to the same class and (ii) they are sufficiently close in the representational space. Test documents are then classified according to the following two-steps procedure: first compute the similarity with each cluster using Rocchio or the Widrow-Hoff rule; then linearly combine these similarities to compute the final label. This procedure provides a significant improvement both over a "pure" $k$-nn approach and a "pure" linear decision.

This paper is organised as follows: in Section 2 we present the Rocchio algorithm and discuss its main advantages and drawbacks. Section 3 explains how this baseline is improved with a weakly supervised clustering procedure. We describe two algorithms which aim at creating such clusters which may prove beneficial for the decision procedure. Section 4 reports and discusses experimental results obtained on 3 different textual databases and Section 5 presents some conclusions and directions for future work. 


\section{Rocchio}

Rocchio is a text classifier originally introduced in [12 to improve information retrieval systems with relevance feedback. It uses the vector space model 13]: every text $d$ is represented by a vector $[d]$ in $R^{n}$ (with $n$ the number of distinct words in the corpus), each coordinate $d_{w}$ can be computed from the frequency $\operatorname{fr}(w, d)$ of word $w$ in $d$ in several manners. One of the most used formulation is :

$$
d_{w}=\operatorname{TFIDF}(w, d)=\log (1+\operatorname{fr}(w, d)) * \log \left(\frac{N}{N(w)}\right)
$$

where $N$ is the number of documents in the corpus and $N(w)$ the number of documents in which $w$ occurs at least once. This formula allocates a higher weight to words which simultaneously occur frequently in the document while occuring only in a few documents. Each vector $[d]$ is then normalized according to: $\underline{d_{w}}=\frac{d_{w}}{\sqrt{\sum_{w} d_{w}^{2}}}$ in $[\underline{d}]$ so as to reduce the distortion caused by the length of documents. A prototypical profile $[c]$ is computed for each class $c$ according to :

$$
c_{w}=\frac{t}{N_{c}} \sum_{d \in c} \frac{d_{w}}{N_{\bar{c}}} \sum_{d \notin c} \frac{1-t}{d_{w}}
$$

with $N_{c}$ the number of documents in $c, N_{\bar{c}}$ the number of documents not in $c$ and $t$ a free parameter between 0 and 1 . These profiles are defined as the centroid of the examples (with a positive coefficient for examples in the class and a negative one for the others). These vectors are also normalized. In the context of a routing task, $t=1$ is a usual choice, meaning that in this case, negative examples do not contribute to the centroids.

Classification of new documents is performed by computing the euclidian distance between the document vector and the prototype vector of each class; the document is then assigned to the nearest class. As all vectors (prototypes and documents) are normalized, euclidian distance is equivalent to cosine similarity or dot product, which is used for implementation reasons.

In the context of this paper, Rocchio exhibits two important characteristics :

- The decision rule computed by Rocchio is a linear separator (hyperplane) in the vector space. It gives Rocchio the same expressivity as a Perceptron classifier. As [8] shows on filtering tasks, the accuracy of Rocchio with dynamic feedback is comparable to that of a neural network trained with a gradient descent algorithm.

- The learning model of Rocchio is a generative one. Parameters are optimized to match the data, not to discriminate the different classes. Generative models usually have lower asymptotic performance value than discriminative ones, but they converge to their optimal performance faster, especially when there is lots of parameters to estimate (which is the case in the textual classification domain) [10].

We have shown in 19 that Rocchio is robust in the presence of noise and very effective for routing tasks with a high number of classes. On the other 
hand, its inability to take into account the substructure of classes (such as different subtopics) is a significant drawback compared to others approaches. Rocchio vastly underperforms other algorithms when classes contain intermixed subtopics: when some subtopics of different classes are nearer than subtopics of the same class (for example in the case of spam filtering, the subtopic on software advertisement can be closer to legitimate emails than to other subtopics of spams such as ones with pornographic content). In the remainder of this paper, we will call classes with such intermixed subtopics "heterogeneous classes". Figure 1 shows an example of homogeneous and heterogeneous classes in terms of relative positioning of subtopics in the vector space.

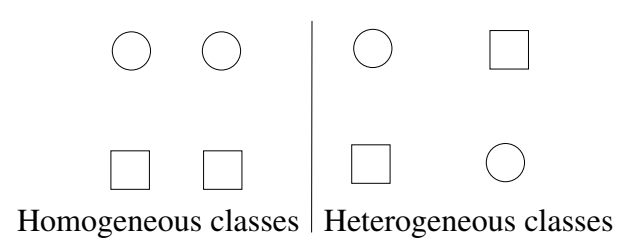

Fig. 1. Homogeneous and heterogeneous corpus (round and square classes have two subtopics).

These difficulties in dealing with classes with intermixed subtopics come from the generative model used by Rocchio. It assumes that each class has a spherical shape and that the information of the centroid is sufficient to correctly describe a class. Training is accordingly straightforward, because one only has to compute the centroid of all examples for each class. But in cases where the data does not match this model, Rocchio will perform poorly.

To avoid these shortcomings, one need to allow Rocchio to use more complex models while at the same time preserving its simple learning process. This can be achieved with the use of a clustering algorithm which will split the classes into coherent sub-classes. A prototype is then computed for each cluster, as if it were a class in its own right. New examples are labelled according to the class of the nearest prototype. We call this class of algorithms Multi-Prototypes Rocchio (MPR). Even if this procedure can be used with any classifier, it is especially tailored to avoid Rocchio's shortcomings.

Rocchio can be seen as a neural network with no hidden layer. Weights are not learned by a back-propagation algorithm but simply computed as the mean of weights of all examples. Clusters are analog to a hidden layer having as many neurons as clusters. Weights of connection between initial layer and hidden layer are still computed by the mean of examples, weights between the hidden layer and the final layer are binary values according to the class of the cluster. Propagation of weights are different in MPR, because classification is based on the nearest prototype (the hidden neuron with the highest weight) instead of a weighted sum of all hidden layers. With this analogy, we see that the clustering allows to change the complexity of the underlying model. 
Moreover, preliminary experiments [19] show that the use of subclasses can be very useful for heterogeneous classes but not for homogeneous ones. The model complexity (in other words number of clusters) must not be chosen according to the data but driven by the accuracy of Rocchio. There is no need to separate two subtopics of the same class if they are not mixed with any subtopic of another class. This suggests that clustering must be performed using a discriminative approach rather than a generative one.

Most of the clustering algorithms described in introduction don't meet these requirements, because the clustering process is independent of the categorization one. They don't try to directly optimize the accuracy of the induced classifier (with the exception of the GIS algorithm from Lam).

\section{Clustering Algorithms}

We want to devise a clustering algorithm which detects clusters in heterogeneous classes but not those in homogeneous classes. This condition can be turned into a mathematical criterion in various ways. We have explored two different criteria: the first one is based on the relative positioning of clusters, the second one is more directly error-driven and based on example categorization. Both are integrated into a top-down hierarchical clustering (at each step, we split one cluster into two new smaller clusters). As both criteria uses the class labels of examples, this procedure is not entirely unsupervised, thus the term "weakly supervised".

\subsection{Notations}

Let $\mathcal{C}=\left\{c_{1}, \ldots, c_{k}\right\}$ be a set of $k$ classes, $N$ the number of examples and $\Pi=$ $\left\{\Pi_{1}, \ldots, \Pi_{p}\right\}$ the unknown partition of examples. $c(x)$ is the class of example $x$ and $c\left(\Pi_{i}\right)$ the class labels of the examples in $\Pi_{i}$. Clusters are only allowed to split the existing classes. The partition must then verify:

$$
\forall(x, y) \Pi(x)=\Pi(y) \Rightarrow c(x)=c(y)
$$

\subsection{Top-Down Clustering}

Our algorithm starts with one cluster per class. At each step, we want to split the cluster with the largest dispersion of its documents. Following [5], we measure the dispersion with the square-root of the average pairwise similarity between documents:

$$
\begin{aligned}
\|c\|^{2} & =\sum_{w} c_{w}^{2}=\frac{1}{N_{c}^{2}} \sum_{w}\left(\sum_{d \in c} d_{w}\right)^{2} \\
& =\frac{1}{N_{c}^{2}} \sum_{w}\left(\sum_{d 1, d 2 \in c} d 1_{w} d 2_{w}\right) \\
& =\frac{1}{N_{c}^{2}} \sum_{d 1, d 2 \in c}[d 1][d 2]
\end{aligned}
$$

So we choose the cluster with the smallest norm and apply any clustering algorithm to create two subclusters. We choose K-Means for its simplicity. The resulting split is tested against the criterion. If the division is not accepted, we try with the cluster with the second smallest norm and so on until no more split is accepted. 


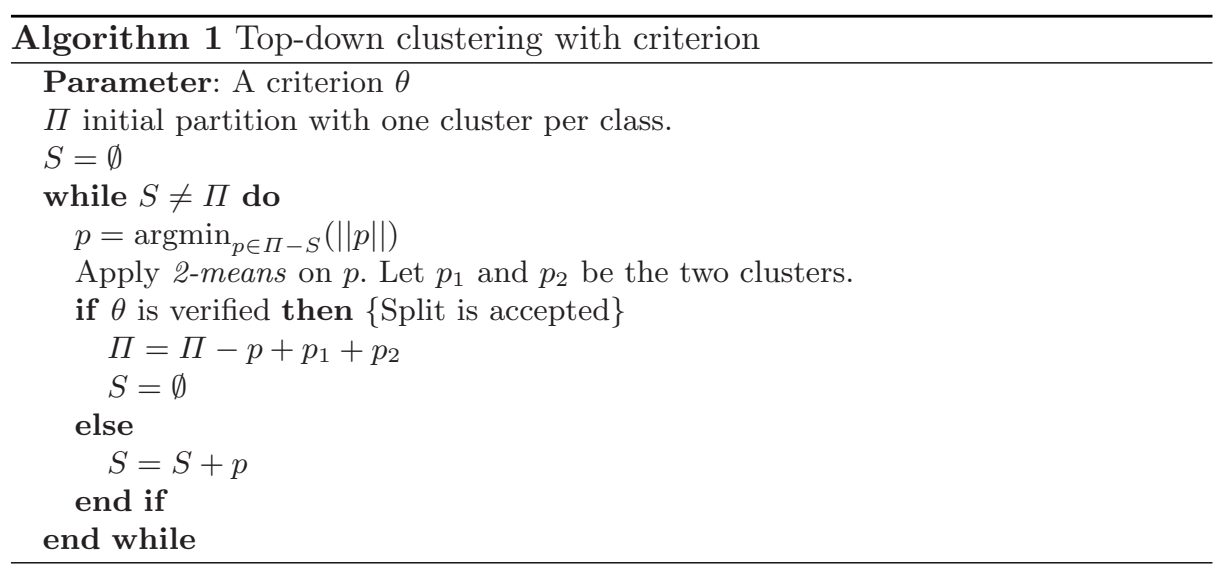

\subsection{Criterion RP: With Relative Positioning of Clusters}

$$
\forall i, j c\left(\Pi_{i}\right)=c\left(\Pi_{j}\right) \Rightarrow \exists k, c\left(\Pi_{k}\right) \neq c\left(\Pi_{i}\right) \text { and }\left\{\begin{array}{l}
d\left(\Pi_{i}, \Pi_{k}\right) \leq d\left(\Pi_{i}, \Pi_{j}\right) \\
d\left(\Pi_{j}, \Pi_{k}\right) \leq d\left(\Pi_{i}, \Pi_{j}\right)
\end{array}\right.
$$

This constraint means that for each pair of clusters of the same class, there must exist a third cluster of another class between the two (see figure 2 for a small illustration). This constraint is obviously satisfied with one cluster per class. The criterion we used aims at maximizing the number of clusters, while keeping the constraint satisfied.

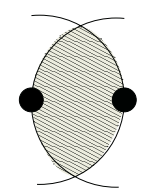

Fig. 2. Illustration of the "zone between two clusters".

Given the highly combinatorial nature of the problem, we have used a approximation, which relies on a greedy algorithm. At each step of the clustering process, we only test if the two newly constructed clusters verify our criterion. The global constraint is not garanteed because we do not verify it for all the other pairs of clusters.

An additional threshold controls the clusters size, preventing to built too small solutions, which are often noisy and unreliable : clusters are never accepted if they contain less than $N_{0}$ examples. Without this filtering, the algorithm will create noisy clusters covering very few examples. These clusters will then allow more splits to be accepted. In our experiments, we have found that without filtering, the algorithm finishes with lots of very small clusters which is clearly not what we want. We have chosen $N_{0}=20$ for all our experiments. 


\subsection{Criterion $D C$ : With Training Documents Categorization}

$$
\operatorname{Min} \mid\left\{x \mid \exists \Pi_{1}, \Pi_{2} \text { s.t. } \begin{array}{l}
c\left(\Pi_{1}\right)=c\left(\Pi_{2}\right)=c(x) \\
\Pi_{1}, \Pi_{2} \text { nearest clusters of } x
\end{array}\right\} \mid
$$

This formula expresses the fact that we want to minimize the number of examples for which the two nearest prototypes are in the same class. The idea is that if this is true for a lots of examples, then we don't need to distinguish those two prototypes. As before, this formula is obviously minimal with one cluster per class. Whenever a second cluster is created for any class, there is almost always at least one example which does not satisfy the criterion anymore. The constraint (6) has been relaxed as follows: new clusters are accepoted if less than $m * \min \left(\left|p_{1}\right|,\left|p_{2}\right|\right)$ examples of the two clusters do not satisfy the constraint anymore, with $m$ a free parameter. Experiments show that results are not very sensitive to the value of $m$ (we have tried from $m=0.5$ to $m=5$ with no significant differences).

Finally, we also need to point out that this constraint only take into account correctly classified examples, which implicitely lowers the influence of noisy examples. This explains why there is no need to filter small clusters, filtering being already implicitely performed by the criterion.

\section{Experiments and Results}

\subsection{Corpora}

We have used three different corpora: Newsgroups, Spam and Mail Center.

The Newsgroups corpus, collected by Ken Lang, contains 20000 messages, evenly distributed in 20 classes, each class corresponding to a different Usenet newsgroup 1. A list of Newsgroups is given in table 1 Two new corpora were then derived: the first one is obtained by merging newsgroups with the same Usenet prefix 2 , leading to a partition of messages into 4 homogeneous classes; the second one results from the merging of unrelated newsgroups into four superclasses, so as to have intermixed subclusters. This corpus is later referred as the heterogeneous corpus.

The Spam corpus contains 2193 emails. The task is here to discriminate junk or unsollicited emails from the legitimate ones. The corpus contains 1460 spams for 733 legitimate emails. Messages in English and in French are mixed in the two classes.

The Mail Center corpus contains 2393 emails received by a customer service classified in 40 categories (see [18] for more information regarding this corpus). Messages are mostly written in French.

In all our experiments, two third of the corpus is used for training and the remaining part is used for testing.

\footnotetext{
1 The corpus can be downloaded at http://www-2.cs.cmu.edu/afs/cs/project/theo-20/www/data/news20.html

${ }^{2}$ We have placed soc.religion.christian, alt.atheism and misc.forsale in the most similar classes so as to have super-classes with homogeneous sizes.
} 
Table 1. List of groups for the newsgroups corpus.

1. comp.graphics

2. comp.windows.x

11. alt.atheism

3. comp-os.ms-windows.misc

4. comp.sys.mac.hardware

5. comp.sys.ibm.pc.hardware

6. talk.politics.guns

7. talk.politics.mideast

8. talk.politics.misc

9. talk.religion.misc

10. soc.religion.christian
12. sci.electronics

13. sci.crypt

14. sci.space

15. sci.med

16. misc.forsale

17. rec.sport.baseball

18. rec.sport.hockey

19. rec.autos

20. rec.motorcycles
Base 1: non mixed subclusters

C1 : $1,2,3,4,5$

C2 : $6,7,8,9,10,11$

C3 : $12,13,14,15$

C4 : $16,17,18,19,20$

Base 2: Intermixed subclusters

C1 : 1,2,6,12,16

C2 : $3,7,8,13,17$

C3 : $4,9,10,14,18$

C4 : $5,11,15,19,20$

Table 2. Performance measure: Acc-1/Acc-2. Bold values show the best algorithm for each corpus. There is no Acc-2 measure for the Spam corpus since it contains only two classes.

\begin{tabular}{|c|c|c|c|c|c|}
\hline & \multicolumn{3}{|c|}{ Newsgroups } & \multirow[t]{2}{*}{ Spam } & \multirow{2}{*}{$\begin{array}{c}\text { Mail } \\
\text { Center }\end{array}$} \\
\hline & normal & heterogeneous & homogeneous & & \\
\hline Rocchio & $0.810 / 0.921$ & $0.754 / 0.921$ & $0.892 / 0.973$ & $0.771 /$. & $0.508 / 0.724$ \\
\hline $\mathrm{K}-\mathrm{NN}$ & $0.844 / \mathbf{0 . 9 4 4}$ & $0.864 / \mathbf{0 . 9 7 0}$ & $0.932 / 0.983$ & $0.930 /$. & $0.535 / 0.649$ \\
\hline SVM & $\mathbf{0 . 8 6 5} / 0.932$ & $\mathbf{0 . 8 9 0 / 0 . 9 6 8}$ & $0.959 / 0.990$ & $0.974 /$ & $0.578 / 0.713$ \\
\hline clustered-SVM & & $0.878 / 0.960$ & $0.940 / 0.984$ & $0.978 /$. & $0.532 / 0.698$ \\
\hline K-Means & $0.810 / 0.921$ & $0.815 / 0.944$ & $0.904 / 0.973$ & $0.959 /$. & $0.527 / 0.726$ \\
\hline Greedy clustering & $0.809 / 0.921$ & $0.804 / 0.943$ & $0.908 / 0.975$ & $0.948 /$. & $0.560 / 0.729$ \\
\hline Criterion RP & $0.813 / 0.924$ & $0.818 / 0.942$ & $0.905 / 0.972$ & $0.964 /$. & $0.522 / 0.723$ \\
\hline Criterion DC & $0.818 / 0.922$ & $0.813 / 0.932$ & $0.907 / 0.973$ & $0.962 /$. & $0.562 / \mathbf{0 . 7 3 1}$ \\
\hline
\end{tabular}

\subsection{Accuracy Measures}

Experiments have been performed for all corpora with the following algorithms: $K$ nearest neighbors, Support Vector Machine, simple Rocchio, Clustered-SVM, MPR with $K$-Means, criterion-based clustering and a greedy division clustering. The greedy clustering always splits the cluster with the smallest norm. ClusteredSVM is similar to the work of [11]: after clustering, a SVM is learned for each cluster and new documents are assigned to the class of the best cluster. For algorithms where the number of clusters must be provided (such as $K$-Means), we have used the number found by criterion-based clustering. To compare algorithms, we have used a more general performance measure than usual accuracy. Acc- $n$ is defined as the pourcentage of test examples for which the correct class is found in one of the $n$-best answers of the algorithm. This measure seems to provide a reasonable estimate of actual performance in applicative contexts involving a human validation [18. We have shown in 19 that Rocchio performs comparatively much better with Acc- $n(n>1)$ than with Acc-1. 
Results presented in table 2 suggest the following comments:

- SVM constantly outperforms all other algorithms for the Acc-1 measure.

- Clustered-SVM is always lower than simple SVM, as in [11. This confirms that clustering is useful for Rocchio but not necessarily for other classifiers.

- For the three newsgroups corpora, MPR is only slightly better than Rocchio and always worse than k-NN or SVM.

- For Spam and Mail Center databases, the improvement over Rocchio is very sensible and MPR surpasses k-NN and even SVM for Mail Center with Acc-2.

- MPR improves Rocchio more for Acc-1 than for Acc-2.

- The different clustering algorithms have a very similar behaviour. The main advantage of our criterion-based clustering is its ability to automatically determine the "right" number of clusters. Overall, the DC criterion is more stable and accurate and is used for the rest of our experiments.

\subsection{Discussions about Number of Clusters Found by MPR}

Both implementations of MPR are also able to create useful clusters to improve overall accuracy. Unlike $K$-Means or any unsupervised clustering, they are able to choose the right number of clusters.

This fact is confirmed by an examination of the results on the Newsgroups homogeneous and heterogeneous corpora. For this experiments, the right number of clusters is known in advance: for the homogeneous corpus we expect clustering to be useless, whereas for the heterogeneous, we expect to get better results by recovering the 20 initial classes. Experimental results are reported in table 3 Obviously, MPR is able to see a difference between these two simulated corpora and to pick up an approximately correct number of clusters.

Table 3. Number of clusters found by each algorithm.

\begin{tabular}{|c|c|c|}
\hline & homogeneous & heterogeneous \\
\hline Optimal & 4 or 20 & 20 \\
Criterion RP & $6.2(0.905)$ & $22(0.818)$ \\
Criterion DC & $5.4(0.905)$ & $14(0.813)$ \\
\hline
\end{tabular}

A second way to check that the number of clusters is correct is to do an exhaustive search. To this end, we have used the greedy clustering with an increasing number of clusters. Each of these is then integrated in the classifier. Results are presented on Figure 3. Our criterion seems to always find just a little less clusters than the optimum value, the resulting accuracy being slightly lower than the maximum found by greedy clustering. Overall, we think that the criterion-based clustering is able to find an appropriate number of clusters. 


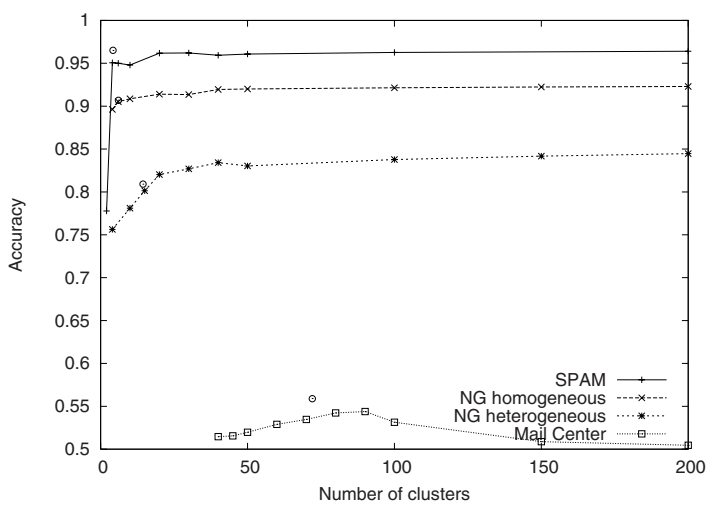

Fig. 3. Accuracy with increasing numbers of clusters. The dots represent number of clusters and accuracy for MPR with criterion DC.
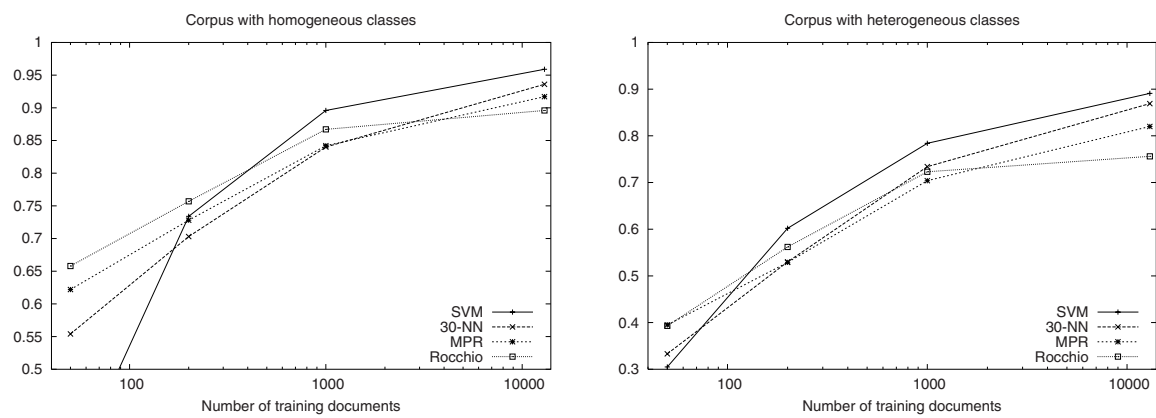

Fig. 4. Accuracy according to the number of learning examples.

\subsection{Influence of Corpus Size}

Rocchio is very efficient and often outperforms other algorithms when the training corpus contains very few examples per class. The clustering algorithms presented here require an important number of examples to be able to create statistically coherent clusters. To see how the combination of clustering and Rocchio behaves on a small corpus, we have performed additional experiments with corpora of varying size.

Results are reported on Figure 4. As expected, Rocchio outperforms all others classifiers with fery few documents but its learning curve rapidly flattens. MPR is a compromise between Rocchio and k-NN / SVM with better accuracy than k-NN or SVM with few examples and better than Rocchio with lots of examples.

\section{Conclusion and Future Work}

We have presented here a weakly supervised clustering algorithm and demonstrate the usefulness of this learning procedure when used in conjonction with 
a Rocchio classifier for text categorization tasks. Unlike related work, this algorithm tries to use the supervision data (class labels) to guide clustering. We think this is the main reason for the difference of results between [11] and our work. This strategy provides the clustering algorithm with a well-behaved stopping criterion, which allows to automatically discover the right number of clusters. The criterion based on performance measures seems to be the most accurate and most stable one. Using this weakly supervised clustering, we have successfully managed to improve Rocchio's performance, with errors rate dropping between $4 \%$ and $84 \%$ depending on the corpus. These differences in performance confirms that Rocchio can use some extra information regarding the internal organization of clusters, but this information is not useful for all corpora. In our experiments, we found that the number of useful subclusters is relatively small, thus preserving the efficiency of Rocchio during the classification phase. We have also identified an important characteristic of our clustering algorithm: it requires more documents than a simple Rocchio classifier; in fact using it with a too small corpus can even lower accuracy.

Our clustering algorithm allows to discover some hidden structure on any textual database in a way that is beneficial for the classifier Rocchio. We plan to investigate the use of similar unsupervised clustering techniques for other tasks including forgetting of past examples after a concept shift and management of a temporal stream of documents by monitoring these clusters.

\section{References}

1. Chris Buckley and Gerard Salton. Optimization of relevance weights. In Proceedings of the Eighteenth Annual International ACM SIGIR Conference of Research and Development in Information Retrieval, pages 351-357, 1995.

2. P. Cheeseman, J. Kelly, M. Self, J. Stutz, W. Taylor, and D. Freeman. Autoclass: A bayesian classification system. In Proceedings of the Fifth International Conference on Machine Learning, pages 54-64, Ann Arbor, June 1988. Morgan Kaufmann Publishers.

3. H. de Kroon, T. Mitchell, and E. Kerckhoffs. Improving learning accuracy in information filtering. In International Conference on Machine Learning - Workshop on Machine Learning Meets HCI, 1996.

4. D. Eichmann, M. Ruiz, P. Srinivasan, N. Street, C. Chris, and F. Menczer. A cluster based approach to tracking, detection and segmentation of broadcast news. In Proceedings of the DARPA Broadcast News Workshop, pages 69-76, 1999.

5. Eui-Hong Han and George Karypis. Centroid-based document classification: Analysis and experimental results. In Principles of Data Mining and Knowledge Discovery, pages 424-431, 2000.

6. Thorsten Joachims. Text categorization with support vector machines: Learning with many relevant features. In ECML-98, Tenth European Conference on Machine Learning, pages 137-142, 1998.

7. Wai Lam. Using a generalized instance set for automatic text categorization. In Proceedings of SIGIR-98, 21th ACM International Conference on Research and Development in Information Retrieval, pages 81-89, 1998. 
8. David D. Lewis, Robert E. Schapire, James P. Callan, and Ron Papka. Training algorithms for linear text classifiers. In Proceedings of SIGIR-96, pages 298-306, Zürich, CH, 1996.

9. Alessandro Moschitti. A study on optimal parameter tuning for rocchio text classifier. In proceedings of the 25th European Conference on Information Retrieval Research (ECIR), Pisa, Italy, 2003.

10. Andrew Y. Ng and Michael I. Jordan. On discriminative vs. generative classifiers: A comparison of logistic regression and naive bayes. Neural Information Processing Systems, 2001.

11. J-H Oh, K-S Lee, D-S Chang, C. Won Seo, and K-S Choi. Trec-10 experiments at kaist: Batch filtering and question answering. In Proceedings of The Tenth Text REtrieval Conference (TREC-10), pages 347-354, 2001.

12. Joseph John Rocchio. The SMART Retrieval System: Experiments in Automatic Document Processing, chapter 14, Relevance Feedback in Information Retrieval, pages 313-323. Gerard Salton (editor), Prentice-Hall Inc., New Jersey, 1971.

13. Gerard Salton, A. Wong, and C.S. Yang. A vector space model for information retrieval. Communications of the ACM, 18(11):613-620, November 1975.

14. Robert E. Schapire and Yoram Singer. BoosTexter: A boosting system for text classification. Machine Learning, 39(2/3):135-168, 2000.

15. Robert E. Schapire, Yoram Singer, and Amit Singhal. Boosting and rocchio applied to text filtering. In W. Bruce Croft, Alistair Moffat, Cornelis J. van Rijsbergen, Ross Wilkinson, and Justin Zobel, editors, Proceedings of SIGIR-98, 21st ACM International Conference on Research and Development in Information Retrieval, pages 215-223, Melbourne, AU, 1998. ACM Press, New York, US.

16. Fabrizio Sebastiani. Machine learning in automated text categorization. $A C M$ Computing Surveys, 34(1):1-47, 2002.

17. Amit Singhal, Mandar Mitra, and Christopher Buckley. Learning routing queries in a query zone. In Proceedings of SIGIR-97, 20th ACM International Conference on Research and Development in Information Retrieval, pages 25-32, Philadelphia, US, 1997.

18. Romain Vinot and François Yvon. Semi-automatic response in a Mail Center. In ASMDA 2001, 10th International Symposium on Applied Stochastic Models and Data Analysis, pages 992-997. Université de Technologie de Compiègne, 2001.

19. Romain Vinot and François Yvon. Quand simplicité rime avec efficacité : Analyse d'un catégoriseur de textes. In Colloque International sur la Fouille de Texte (CIFT'02), pages 17-26, Tunisie, 2002.

20. Yiming Yang. An evaluation of statistical approach to text categorization. Journal of Information Retrieval, 1(1/2):67-88, 1999. 